\title{
Preface: Critical Concepts in the Oncogenesis of Adenocarcinoma of the Rectum
}

Tumor phenotype is currently being used in the management of breast cancer. Assays such as Onocotype DX and MammaPrint are in clinical use to guide treatment options in the management of breast cancer. ${ }^{1}$ In colon cancer, we have learned that patients with mutations of KRAS will not respond to anti-EGFR therapy. These are specific examples that underscore the fact that the idea of "one size fits all" in the management of cancer must be eliminated. The preoperative management of stage II and III rectal cancer presents a specific problem that exemplifies this issue. On one side of the spectrum, some patients with rectal cancer achieve a complete obliteration of the tumor following preoperative chemoradiation. However, up to one third of patients treated similarly do not respond to the same form of management. Dr. William and Dr. Meyer explore the current guidelines in the delivery and schedule of ionizing radiation in patients with rectal cancer and discuss the areas of improvement. These are currently considered the mainstays in the ability to reduce tumor burden in patients with rectal cancer.

The management of a tumor based on its phenotype is becoming common practice. Similarly, a multimodal approach for cancer treatment is not only logical, but a necessity that must be adopted in chemoradioresistant tumors. Multimodal treatment takes advantage of the ability to use numerous interventions to minimize the dose of a single agent, which minimizes adverse effects while maximizing tumor cell death. Dr. Illum presents the current chemosensitizing strategies for the management of rectal cancer. Although there have been multiple attempts to maximize our current overall success, these have been challenging, as discussed by many manuscripts in this issue of the journal.
Because of the emphasis of individualized treatment, predictive biomarkers need to be developed to the level of sophistication to both be practical and cost effective in clinical practice. Dr. Kapur discusses the current status of biomarkers that could be utilized to predict a response to chemoradiation. Dr. Kapur also explains the limitation of pathological examinations to determine an appropriate response to chemoradiation. Both of these issues are important in setting the next era of research in the management of rectal cancer. In spite of the drastic revolution that has occurred in the preoperative management of rectal cancer, the mainstay of treatment remains surgical intervention. This area has also evolved, and alternative surgical options have been described and are summarized by Dr. Olson. The evolving management of rectal cancer mandates a detailed understanding of the molecular changes and pathways that characterize chemoradioresistant tumors. In order to develop biomarkers and predictive molecules to assist with this malignancy, the biology of rectal tumors must be well understood. This biology can also be utilized for targeted therapy, an evolving area of rectal cancer discussed by Dr. Dineen.

The obstacles to be overcome are paramount, but the current directions point to a bright horizon. Multiple areas are being targeted for improvement in the management of rectal cancer and this is explored in the last manuscript of this special issue of the journal. I thank all of the contributing authors for their expertise reviewed in their manuscripts. 


\section{REFERENCES}

1. Kim C, Paik S. Gene-expression-based prognostic assays for breast cancer. Nat Rev Clin Oncol. 2010;7:340-7.
Guest Editor:

Sergio Huerta, M.D., F.A.C.S.

Assistant Professor of Surgery

University of Texas Southwestern Medical Center Dallas, Texas 75225 